\title{
Bases of exponents with a piecewise linear phase in generalized weighted Lebesgue space
}

\author{
Tofig Najafov', Natavan Nasibova ${ }^{2 *}$ and Zahira Mamedova ${ }^{3}$
}

\section{"Correspondence:}

natavan2008@gmail.com

${ }^{2}$ Department of Non-Harmonic

Analysis, Institute of Mathematics

and Mechanics of NAS of

Azerbaijan, 9 B. Vahabzadeh Str.,

Baku, 1141, Azerbaijan

Full list of author information is

available at the end of the article

\begin{abstract}
The perturbed system of exponents with a piecewise linear phase, consisting of eigenfunctions of a discontinuous differential operator, is considered in this work. Under certain conditions on the weight function of the form of a power function, sufficient conditions for the basicity of this system are obtained in generalized weighted Lebesgue space.
\end{abstract}

Keywords: system of exponents; weighted space; variable exponent; basicity

\section{Introduction}

The perturbed system of exponents $\left\{e^{i \lambda_{n} t}\right\}_{n \in Z}$ plays an important role in the study of spectral properties of discrete differential operators and in the approximation theory. Apparently, the study of basis properties (completeness, minimality, basicity) of these systems dates back to the well-known work of Paley and Wiener [1]. Since then, a lot of research has been made in this field (more details can be found in [2-8]). It should be noted that similar systems are of great scientific interest in the frame theory as well, which dates back to the seminal paper by Duffin and Schaeffer [9]. More details as regards these and related facts can be found in $[7,9,10]$.

Since recently, there arose a great interest in considering various problems, related to some research fields of mechanics and mathematical physics, in generalized Lebesgue spaces $L_{p(\cdot)}$ with a variable summability exponent $p(\cdot)$. Some fundamental results of classical harmonic analysis were carried over to the case of $L_{p_{(\cdot)}}$. More details as regards these facts can be found in [11-16]. It should be noted that the application of the Fourier method to the problems for partial differential equations in generalized Sobolev classes requires a good knowledge of the approximative properties of perturbed exponential systems in generalized Lebesgue spaces. Approximation-related issues in these spaces have been first studied by Sharapudinov (see e.g. [14]).

A system of exponents with a piecewise linear phase is considered in this paper. When the weight of the form of power function, the basis properties of this system are studied in a weighted space $L_{p_{(\cdot)}, \rho}$. when the weight has a power form. Sufficient conditions for completeness, minimality and basicity are obtained in $L_{p_{(-)}, \rho}$. It should be noted that the basis properties of exponential systems with a linear phase in $L_{p(\cdot)}$ have been previously studied in [17-19].

(c) 2016 Najafov et al. This article is distributed under the terms of the Creative Commons Attribution 4.0 International License (http://creativecommons.org/licenses/by/4.0/), which permits unrestricted use, distribution, and reproduction in any medium, provided you give appropriate credit to the original author(s) and the source, provide a link to the Creative Commons license, and indicate if changes were made. 


\section{Background information}

We will use the usual notations. $N$ will be a set of all positive integers; $Z$ will be a set of all integers; $Z_{+}=\{0\} \cup N ; R$ will be the set of all real numbers; $C$ will stand for the field of complex numbers; $(\cdot)$ is the complex conjugate; $\delta_{n k}$ is the Kronecker symbol; $\chi_{A}(\cdot)$ is the characteristic function of the set $A$.

Let us present some facts from the theory of Lebesgue spaces with a variable summability exponent. Let $p:[-\pi, \pi] \rightarrow[1,+\infty)$ be some Lebesgue measurable function. By $\mathrm{L}_{0}$ denote the class of all functions measurable on $[-\pi, \pi]$ (with respect to Lebesgue measure). Denote

$$
I_{p}(f) \stackrel{\text { def }}{=} \int_{-\pi}^{\pi}|f(t)|^{p(t)} d t .
$$

Let

$$
\mathrm{L} \equiv\left\{f \in \mathrm{L}_{0}: I_{p}(f)<+\infty\right\}
$$

With respect to the usual linear operations of addition and multiplication by a number, $\mathrm{L}$ is a linear space as $p^{+}=\sup \operatorname{vrai}_{[-\pi, \pi]} p(t)<+\infty$. With respect to the norm

$$
\|f\|_{p(\cdot)} \stackrel{\text { def }}{=} \inf \left\{\lambda>0: I_{p}\left(\frac{f}{\lambda}\right) \leq 1\right\}
$$

$\mathrm{L}$ is a Banach space (see e.g. [12]), and we denote it by $L_{p(\cdot) .}$. Let

$$
\begin{aligned}
W L \stackrel{\text { def }}{=}\left\{p: p(-\pi)=p(\pi) ; \exists C>0, \forall t_{1}, t_{2} \in[-\pi, \pi]:\right. \\
\left.\quad\left|t_{1}-t_{2}\right| \leq \frac{1}{2} \Rightarrow\left|p\left(t_{1}\right)-p\left(t_{2}\right)\right| \leq \frac{C}{-\ln \left|t_{1}-t_{2}\right|}\right\} .
\end{aligned}
$$

Throughout this paper $q(\cdot)$ will denote the conjugate of a function $p(\cdot): \frac{1}{p(t)}+\frac{1}{q(t)} \equiv 1$. Denote $p^{-}=\inf \operatorname{vrai}_{[-\pi, \pi]} p(t)$. The following generalized Hölder inequality is true

$$
\int_{-\pi}^{\pi}|f(t) g(t)| d t \leq c\left(p^{-} ; p^{+}\right)\|f\|_{p(\cdot)}\|g\|_{q(\cdot)},
$$

where $c\left(p^{-} ; p^{+}\right)=1+\frac{1}{p^{-}}-\frac{1}{p^{+}}$. Directly from the definition we get the property which will be used in sequel.

Property A If $|f(t)| \leq|g(t)|$ a.e. on $(-\pi, \pi)$, then $\|f\|_{p_{(\cdot)}} \leq\|g\|_{p_{(\cdot)}}$.

It is easy to prove the following.

Statement 1 Let $p \in W L, p(t)>0, \forall t \in[-\pi, \pi] ;\left\{\alpha_{i}\right\}_{0}^{m} \subset R$. The weight function

$$
\rho(t)=|t|^{\alpha_{0}} \prod_{i=1}^{m}\left|t-\tau_{i}\right|^{\alpha_{i}},
$$


belongs to the space $L_{p(\cdot)}$, if the following inequalities:

$$
\alpha_{i}>-\frac{1}{p\left(\tau_{i}\right)}, \quad \forall i=\overline{0, m}
$$

are satisfied, where $-\pi=\tau_{1}<\tau_{2}<\cdots<\tau_{m}=\pi, \tau_{0}=0, \tau_{i} \neq 0, \forall i=\overline{1, m}$.

The following fact plays an important role in obtaining the main results (see e.g. [12]).

Property B If $p(t): 1<p^{-} \leq p^{+}<+\infty$, then the class $C_{0}^{\infty}(-\pi, \pi)$ (class of finite and indefinitely differentiable functions) is everywhere dense in $L_{p(\cdot)}$.

By $S$ we denote the singular integral

$$
S f=\frac{1}{2 \pi i} \int_{\Gamma} \frac{f(\tau)}{\tau-t} d \tau, \quad t \in \Gamma
$$

where $\Gamma \subset C$ is some piecewise Hölder curve on $C$. Define the weight class $L_{p(\cdot), \rho(\cdot)}$ :

$$
L_{p(\cdot), \rho(\cdot)} \stackrel{\text { def }}{=}\left\{f: \rho f \in L_{p(\cdot)}\right\}
$$

furnished with a norm $\|f\|_{p(\cdot), \rho(\cdot)} \stackrel{\text { def }}{=}\|\rho f\|_{p(\cdot)}$. The validity of the following statement is established in [16].

Statement 2 Let $p \in W L, 1<p^{-}$. Then the singular operator $S$ is acting boundedly from $L_{p(\cdot), \rho(\cdot)}$ to $L_{p(\cdot), \rho(\cdot)}$ if and only if

$$
-\frac{1}{p\left(\tau_{k}\right)}<\alpha_{k}<\frac{1}{q\left(\tau_{k}\right)}, \quad k=\overline{0, m}
$$

Define the generalized weighted Hardy classes $H_{p(\cdot), \rho}^{ \pm}$. By $H_{p_{0}}^{+}$we denote the usual Hardy class, where $p_{0} \in[1,+\infty)$ is some number. Define $H_{p(\cdot), \rho}^{ \pm} \equiv\left\{f \in H_{1}^{+}: f^{+} \in L_{p(\cdot), \rho}(\partial \omega)\right\}$, where $\omega=\{z \in C:|z|<1\}$ and $f^{+}$are non-tangential boundary values on $\partial \omega$ of $f$.

We will need the following theorem from [20].

Theorem 1 Let $p \in W L, p^{-}>1$, and let the inequalities (1) be satisfied. Then, if $F \in H_{p(\cdot), \rho^{+}}$, then $F^{+} \in L_{p(\cdot), \rho}$ :

$$
F(z)=\frac{1}{2 \pi} \int_{-\pi}^{\pi} K_{z}(t) F^{+}(t) d t
$$

where $K_{z}(t) \equiv \frac{1}{1-z e^{-i t}}$ is a Cauchy kernel. Vice versa, if $F^{+} \in L_{p(\cdot), \rho}$, then the function $F$, defined by (2), belongs to the class $H_{p(\cdot), \rho}^{+}$, where $F^{+}$are non-tangential boundary values of $F(\cdot)$ on $\partial \omega$.

The weighted Hardy class ${ }_{m} H_{p(\cdot), \rho}^{-}$of functions which are analytic in $C \backslash \bar{\omega}(\bar{\omega}=\omega \cup \partial \omega)$ with their orders $m_{0} \leq m$ at infinity is defined similarly to the classical one. Let $f(z)$ be the 
analytic function in $C \backslash \bar{\omega}$ of finite order $m_{0} \leq m$ at infinity, i.e.

$$
f(z)=f_{1}(z)+f_{2}(z)
$$

where $f_{1}(\cdot)$ is a polynomial of degree $m_{0} \leq m\left(f_{1}(z) \equiv 0\right.$ for $\left.m_{0}<0\right), f_{2}(\cdot)$ is a regular part of Laurent series expansion of $f(\cdot)$ in the neighborhood of an infinitely remote point. If the function $\varphi(z) \equiv \overline{f_{2}\left(\frac{1}{\bar{z}}\right)}$ belongs to the class $H_{p(\cdot), \rho}^{+}$, then we will say that the function $f(\cdot)$ belongs to the class ${ }_{m} H_{p(\cdot), \rho}^{-}$.

The validity of the following theorem is proved just like in the classical case (see e.g. $[20-22])$.

Theorem 2 Let $p \in W L, p^{-}>1$, and let the inequalities (1) be satisfied. Iff $\in H_{p(\cdot), \rho}^{+}$, then

$$
\left\|f\left(r e^{i t}\right)-f^{+}\left(e^{i t}\right)\right\|_{p(\cdot), \rho} \rightarrow 0, \quad r \rightarrow 1-0,
$$

where $f^{+}$are non-tangential boundary values on $\partial \omega$ off .

A similar result also holds in class ${ }_{m} H_{p(\cdot), \rho}^{-}($see $e . g . ~[20-22])$.

Theorem 3 Let $p \in W L, p^{-}>1$, and let the inequalities (1) be satisfied. If $\in{ }_{m} H_{p(\cdot), \rho}^{-}$, then

$$
\left\|f\left(r e^{i t}\right)-f^{-}\left(e^{i t}\right)\right\|_{p(\cdot), \rho} \rightarrow 0, \quad r \rightarrow 1+0,
$$

where $f^{-}$are non-tangential boundary values on $\partial \omega$ of from the outside of $\omega$.

Let us show the validity an analog of the classical theorem of Smirnov.

Theorem 4 Let $p \in W L, p^{-}>1$, and let the inequalities (1) be satisfied. If $u \in H_{1}^{+}$and $u^{+} \in L_{p(\cdot), \rho}$, then $u \in H_{p(\cdot), \rho}^{+}$.

Indeed, assume that $p \in W L, p^{-}>1$, and let the inequality (1) be fulfilled. Let $u \in H_{1}^{+}$and $u^{+} \in L_{p(\cdot), \rho}$, where $u^{+}$is s non-tangential boundary value on $\partial \omega$ of $u$. Then it is well known that (see e.g. [21])

$$
u(z)=\frac{1}{2 \pi i} \int_{\partial \omega} \frac{u^{+}(\tau)}{\tau-z} d \tau .
$$

Then by Theorem 1 we obtain $u \in H_{p(\cdot), \rho}^{+}$.

In obtaining main results we will essentially use the following results from [22] on the solvability of the Riemann problem in generalized weighted Hardy classes. Consider the following non-homogeneous Riemann problem in the $H_{p(\cdot), \rho}^{+} \times{ }_{m} H_{p(\cdot), \rho}^{-}$classes:

$$
F^{+}(\tau)-G(\tau) F^{-}(\tau)=f(\tau), \quad \tau \in \partial \omega,
$$

where $f \in L_{p(\cdot), \rho}$ is some function. By the solution of problem (3) we mean a pair of analytic functions $\left(F^{+}(z) ; F^{-}(z)\right) \in H_{p(\cdot), \rho}^{+} \times{ }_{m} H_{p(\cdot), \rho}^{-}$boundary values of which satisfy equation (3) almost everywhere on $\partial \omega$.

We will suppose that the coefficient $G(\tau)$ satisfies the following conditions:

(1) $G^{ \pm} \in L_{\infty}(\partial \omega)$;

(2) $\theta(t) \equiv \arg G\left(e^{i t}\right)$ is a piecewise Hölder function on $[-\pi, \pi]$. 
Let $\left\{s_{k}\right\}_{1}^{r}:-\pi<s_{1}<\cdots<s_{r}<\pi$ be the points of discontinuity of the function $\theta(t)$ and

$$
\left\{h_{k}\right\}_{1}^{r}: h_{k}=\theta\left(s_{k}+0\right)-\theta\left(s_{k}-0\right), \quad k=\overline{1, r}
$$

be the corresponding jumps of this function at these points. Let us represent the function $\theta(\cdot)$ in the following form:

$$
\theta(t) \equiv \theta_{0}(t)+\theta_{1}(t), \quad t \in[-\pi, \pi]
$$

where $\theta_{0}(\cdot)$ is a continuous part, and $\theta_{1}(\cdot)$ is a jump function defined by the expression

$$
\theta(-\pi)=0, \quad \theta(t)=\sum_{k: 0<s_{k}<t} h_{k}, \quad t \in[-\pi, \pi] .
$$

Assume

$$
h_{0}=\theta(-\pi)-\theta(\pi) ; \quad h_{0}^{(0)}=\theta_{0}(\pi)-\theta_{0}(-\pi) .
$$

Denote by $\left\{t_{k}\right\}_{0}^{l}:\left\{t_{k}\right\}_{0}^{l} \equiv\left\{\tau_{k}\right\}_{0}^{m} \cup\left\{s_{k}\right\}_{0}^{r}$ the union of sets $\left\{\tau_{k}\right\}_{0}^{m}$ and $\left\{s_{k}\right\}_{0}^{r}$.

Denote by $Z(z)$ the canonical solution of homogeneous problem

$$
F^{+}(\tau)-G(\tau) F^{-}(\tau)=f(\tau), \quad \tau \in \partial \omega,
$$

which is defined by the expressions

$$
Z(z) \equiv Z_{1}(z) Z_{2}(z)
$$

where

$$
Z_{k}(z) \equiv \begin{cases}X_{k}(z), & |z|<1 \\ {\left[X_{k}(z)\right]^{-1},} & |z|>1, k=1,2\end{cases}
$$

and the functions $X_{k}(z)$ are defined by the following integrals with Schwartz kernel:

$$
\begin{aligned}
& X_{1}(z) \equiv \exp \left\{\frac{1}{4 \pi} \int_{-\pi}^{\pi} \ln \left|G\left(e^{i t}\right)\right| \frac{e^{i t}+z}{e^{i t}-z} d t\right\}, \\
& X_{2}(z) \equiv \exp \left\{\frac{i}{4 \pi} \int_{-\pi}^{\pi} \theta(t) \frac{e^{i t}+z}{e^{i t}-z} d t\right\} .
\end{aligned}
$$

Assume

$$
\beta_{k}=\sum_{i=0}^{m} \alpha_{i} \chi_{\left\{t_{k}\right\}}\left(\tau_{i}\right)+\frac{1}{2 \pi} \sum_{i=0}^{r} h_{i} \chi_{\left\{t_{k}\right\}}\left(s_{i}\right), \quad k=\overline{0, l} .
$$

The following theorem is established in $[20,22]$.

Theorem 5 Let $p \in W L, p^{-}>1$, and the inequalities (1) be fulfilled. If the inequalities

$$
-\frac{1}{q\left(t_{k}\right)}<\beta_{k}<\frac{1}{p\left(t_{k}\right)}, \quad k=\overline{0, l}
$$


are true, then the general solution of the Riemann problem (3) in classes $H_{p(\cdot), \rho}^{+} \times{ }_{m} H_{p(\cdot), \rho}^{-}$ can be represented in the following form:

$$
F(z)=P_{m_{0}}(z) Z(z)+F_{1}(z)
$$

where $Z(\cdot)$ is the canonical solution of homogeneous problem, $F_{1}(\cdot)$ is the particular solution of non-homogeneous problem (3) defined by

$$
F_{1}(z)=\frac{Z(z)}{2 \pi} \int_{-\pi}^{\pi} K_{z}(t) Z^{+}\left(e^{i t}\right) f(t) d t
$$

$P_{m_{0}}(\cdot)$ is a polynomial of order $m_{0} \leq m$.

In particular, from this theorem we obtain the following.

Corollary 1 Let all the conditions of Theorem 5 be fulfilled. Then the non-homogeneous Riemann problem (3) is uniquely solvable in classes $H_{p(\cdot), \rho}^{+} \times{ }_{-1} H_{p(\cdot), \rho}^{-}$for $\forall f \in L_{p(\cdot), \rho}$, and this solution can be represented in the form

$$
F(z)=\frac{Z(z)}{2 \pi} \int_{-\pi}^{\pi} K_{z}(t) Z^{+}\left(e^{i t}\right) f(t) d t
$$

where $Z(z)$ is the canonical solution of the corresponding homogeneous problem.

Indeed, in this case $m=-1$ and therefore $P_{m_{0}}(\cdot) \equiv 0$. So, $F_{1}(\infty)=0$, then it is clear that $F_{1}(\cdot) \in{ }_{-1} H_{p(\cdot), \rho}^{-}$, and, as a result, as follows from Theorem $5, F(z) \equiv F_{1}(z)$ is a unique solution of the problem (3).

\section{Main results}

Consider the following system of exponents:

$$
\left\{e_{n}(\cdot)\right\}_{n \in Z}
$$

where

$$
e_{n}(t)=\exp i(n t+\gamma(t) \operatorname{sign} n), \quad \gamma(t)=\alpha t+\beta \operatorname{sign} t
$$

$\alpha, \beta \in R$ are real parameters.

We will study the basicity of the system (4) in $L_{p(\cdot), \rho}$ with respect to the parameters $\alpha$ and $\beta$.

Denote $\gamma_{1}=2 \alpha+\frac{2 \beta}{\pi} ; \gamma_{2}=-\frac{2 \beta}{\pi}$. Consider the following systems:

$$
\begin{aligned}
& h_{n}^{+}(t)=\frac{e^{i(\alpha t+\beta \operatorname{sign} t-2 \beta)}}{2 \pi}\left(e^{i t}+1\right)_{-1}^{-\gamma_{1}}\left(e^{i t}-1\right)_{+1}^{-\gamma_{2}} \sum_{k=0}^{n}(-1)^{n-k} C_{\gamma_{2}}^{n-k} \sum_{s=0}^{k} C_{\gamma_{1}}^{k-s} e^{-s t}, \quad n \in Z_{+}, \\
& h_{n}^{-}(t)=\frac{e^{i(\alpha t+\beta \operatorname{sign} t-2 \beta)}}{2 \pi}\left(e^{i t}+1\right)_{-1}^{-\gamma_{1}}\left(e^{i t}-1\right)_{+1}^{-\gamma_{2}} \sum_{k=1}^{n}(-1)^{m-k} C_{\gamma_{2}}^{m-k} \sum_{s=1}^{k} C_{\gamma_{1}}^{k-s} e^{-s t}, \quad m \in N,
\end{aligned}
$$


where

$$
C_{\gamma}^{n}=\frac{\gamma(\gamma-1) \cdots \gamma(\gamma-n+1)}{n !}, \quad C_{\gamma}^{0}=1,
$$

are the binomial coefficients. The following lemma is true (see e.g. $[2,17])$.

Lemma 1 Let, with respect to the parameters $\alpha, \beta \in R$, the following inequalities be fulfilled:

$$
\left|\alpha+\frac{\beta}{\pi}\right|<\frac{1}{2} ; \quad\left|\frac{\beta}{\pi}\right|<\frac{1}{2} .
$$

Then the following relations hold:

$$
\begin{aligned}
& \left\langle x_{k}^{+}, h_{n}^{+}\right\rangle=\left\langle x_{k+1}^{-}, h_{n+1}^{-}\right\rangle=\delta_{n k}, \quad \forall n, k \in N ; \\
& \left\langle x_{k}^{+}, h_{n+1}^{-}\right\rangle=\left\langle x_{k+1}^{-}, h_{n}^{+}\right\rangle=0, \quad \forall n, k \in N, \\
& x_{n}^{ \pm}=e^{ \pm i[(n-\alpha) t-\beta \operatorname{sign} t]}, \quad \text { where }\langle x, y\rangle=\int_{\pi}^{\pi} x(t) \overline{y(t)} d t .
\end{aligned}
$$

Let us establish the validity of the following lemma.

Lemma 2 Let $p \in W L$ and $p^{-}>1$. If the following inequalities:

$$
\begin{cases}-\frac{1}{p(0)}<\left\{\alpha_{0} ; \alpha_{0}+\gamma_{2}\right\}<\frac{1}{q(0)} ; & -\frac{1}{p(\pi)}<\alpha_{1} ; \\ -\frac{1}{p(\pi)}<\left\{\alpha_{m} ; \alpha_{1}+\gamma_{1}\right\}<\frac{1}{q(\pi)} ; & \alpha_{m}+\gamma_{1}<\frac{1}{q(\pi)} ; \\ -\frac{1}{p\left(\tau_{i}\right)}<\alpha_{i}<\frac{1}{q\left(\tau_{i}\right)}, & i=\frac{2, m-1}{2, m-1}\end{cases}
$$

are fulfilled, then the system of exponents $\left\{e_{n}\right\}_{n \in Z}$ is minimal in $L_{p(\cdot), \rho}$.

Proof Let us determine the conditions under which the inclusions $\left\{x_{n}^{ \pm}\right\} \subset L_{p(\cdot), \rho}$ and $\left\{h_{n}^{ \pm}\right\} \subset L_{q(\cdot), \rho^{-1}}$ are true. It is clear that if $\rho^{p(\cdot)}(\cdot) \in L_{1}$, then $\left\{x_{n}^{ \pm}\right\} \subset L_{p(\cdot), \rho}$. From Statement 1 it follows that under fulfilling the inequalities

$$
\alpha_{i}>-\frac{1}{p\left(\tau_{i}\right)}, \quad \forall i=\overline{0, m}
$$

the inclusion $\rho \in L_{p(\cdot)}$ is true. Consider the inclusion $\left\{h_{n}^{ \pm}\right\} \subset L_{q(\cdot), \rho^{-1}}$. We have

$$
\rho^{-1}(t)=|t|^{-\alpha_{0}} \prod_{i=1}^{m}\left|t-\tau_{i}\right|^{-\alpha_{i}}
$$

It is clear that if $\left|\rho_{0}(x)\right|^{q(x)} \in L_{1}$, then $\left\{h_{n}^{ \pm}\right\} \subset L_{q(\cdot), \rho^{-1}}$, where

$$
\rho_{0}(x)=\left|e^{i x}+1\right|^{-\gamma_{1}}\left|e^{i x}-1\right|^{-\gamma_{2}} \rho^{-1}(x) .
$$

Taking into account the relations

$$
\left|e^{i x}-1\right| \sim\left|\sin \frac{x}{2}\right| \sim|x|, \quad x \in[-\pi, \pi]
$$




$$
\left|e^{i x}+1\right| \sim\left|\sin \frac{x+\pi}{2}\right|\left|\sin \frac{x-\pi}{2}\right| \sim|x-\pi||x+\pi|, \quad x \in[-\pi, \pi],
$$

we get

$$
\rho_{0}(x) \sim|x|^{-\gamma_{2}}|x-\pi|^{-\gamma_{1}}|x+\pi|^{-\gamma_{1}} \rho^{-1}(x), \quad x \in[-\pi, \pi],
$$

where the expression $f \sim g$ on $[-\pi, \pi]$ means that $\exists \delta>0$ :

$$
\delta \leq\left|\frac{f(x)}{g(x)}\right| \leq \delta^{-1}, \quad \forall x \in[-\pi, \pi]
$$

Thus

$$
\rho_{0}(x) \sim|x|^{-\gamma_{2}-\alpha_{0}} \prod_{i=1}^{m}\left|x-\tau_{i}\right|^{-\tilde{\alpha}_{i}}, \quad x \in[-\pi, \pi]
$$

where

$$
\begin{aligned}
& \tilde{\alpha}_{1}=\gamma_{1}+\alpha_{1}, \quad \tilde{\alpha}_{m}=\alpha_{m}+\gamma_{1}, \\
& \tilde{\alpha}_{i}=\alpha_{i}, \quad i=\overline{2, m-1 .}
\end{aligned}
$$

If $\rho \in L_{p(\cdot)}$, i.e. if the inequalities

$$
-\frac{1}{p\left(\tau_{i}\right)}<\alpha_{i}, \quad i=\overline{0, m}
$$

are fulfilled, it is absolutely clear that the inclusion $\left\{x_{k}^{ \pm}\right\} \subset L_{p(\cdot), \rho}$ is true. Let us find the conditions under which the system $\left\{h_{n}^{ \pm}\right\}$belongs to $L_{q(\cdot), \rho^{-1}}$. From the representation for $\left\{h_{n}^{ \pm}\right\}$it directly follows that if $\omega(\cdot) \in L_{q(\cdot), \rho^{-1}}$, then $\left\{h_{n}^{ \pm}\right\} \subset L_{q(\cdot), \rho^{-1}}$, where

$$
\omega(t)=\left|e^{i t}+1\right|^{-\gamma_{1}}\left|e^{i t}-1\right|^{-\gamma_{2}}, \quad t \in[-\pi, \pi] .
$$

We have

$$
\begin{aligned}
& \left|e^{i t}-1\right| \sim|t|, \quad t \in[-\pi, \pi] \\
& \left|e^{i t}+1\right| \sim|t-\pi||t+\pi|, \quad t \in[-\pi, \pi] .
\end{aligned}
$$

\section{Consequently}

$$
\omega(t) \sim|t|^{-\gamma_{2}}|t-\pi|^{-\gamma_{1}}|t+\pi|^{-\gamma_{1}}, \quad t \in[-\pi, \pi] .
$$

It is clear that $\omega \in L_{q(\cdot), \rho^{-1}}$ if and only if $\omega \rho^{-1} \in L_{q(\cdot)}$. The product $\omega \rho$ has the representation

$$
\omega(t) \rho^{-1}(t) \sim|t|^{-\alpha_{0}-\gamma_{2}}|t+\pi|^{-\alpha_{1}-\gamma_{1}}|t-\pi|^{-\alpha_{m}-\gamma_{1}} \prod_{i=2}^{m-1}\left|t-\tau_{i}\right|^{-\alpha_{i}}, \quad t \in[-\pi, \pi] .
$$


Drawing attention to Statement 1, we see that if the inequalities

$$
\begin{array}{ll}
-\frac{1}{q(0)}<-\alpha_{0}-\gamma_{2} ; & -\frac{1}{q(\pi)}<-\alpha_{1}-\gamma_{1} ; \\
-\frac{1}{q(\pi)}<-\alpha_{m}-\gamma_{1} ; & -\frac{1}{q\left(\tau_{i}\right)}<-\alpha_{i}, \quad i=\overline{2, m-1},
\end{array}
$$

are fulfilled, then $\omega \rho^{-1} \in L_{q(\cdot)}$, and as a result, we obtain $\left\{h_{n}^{ \pm}\right\} \subset L_{q(\cdot), \rho^{-1}}$.

Consider the functionals

$$
H_{n}^{ \pm}(f)=\int_{-\pi}^{\pi} f(t) \overline{h_{n}^{ \pm}(t)} d t, \quad f \in L_{p(\cdot), \rho} .
$$

We have

$$
\begin{aligned}
\left|H_{n}^{ \pm}(f)\right| & =\left|\int_{-\pi}^{\pi} f(t) \rho(t) \overline{h_{n}^{ \pm}(t)} \rho^{-1}(t) d t\right| \leq c\left(p^{-} ; p^{+}\right)\|f \rho\|_{L_{p(\cdot)}}\left\|h_{n}^{ \pm} \rho^{-1}\right\|_{L_{q(\cdot)}} \\
& =c\left(p^{-} ; p^{+}\right)\left\|h_{n}^{ \pm} \rho^{-1}\right\|_{L_{q(\cdot)}}\|f\|_{L_{p(\cdot),},} .
\end{aligned}
$$

From this relation it follows directly that if the inclusion $\left\{h_{n}^{ \pm}\right\} \subset L_{q(\cdot), \rho^{-1}}$ is true, then $\left\{H_{n}^{ \pm}\right\} \subset\left(L_{p(\cdot), \rho}\right)^{*}$. Taking into account Lemma 1 we get the validity of the lemma.

The following main theorem is true.

Theorem 6 Let $p \in W L, p^{-}>1$ and the following inequalities be satisfied:

$$
\begin{aligned}
& -\frac{1}{p\left(\tau_{i}\right)}<\alpha_{i}<\frac{1}{q\left(\tau_{i}\right)}, \quad i=\overline{0, m} ; \\
& -\frac{1}{p(0)}<\alpha_{0}+\gamma_{2}<\frac{1}{q(0)} ; \quad-\frac{1}{p(\pi)}<\alpha_{1}+\gamma_{1}<\frac{1}{q(\pi)} ; \\
& -\frac{1}{p(\pi)}<\alpha_{m}+\gamma_{1}<\frac{1}{q(\pi)} ; \quad \gamma_{1}=2\left(\alpha+\frac{\beta}{\pi}\right) ; \quad \gamma_{2}=-\frac{2 \beta}{\pi} .
\end{aligned}
$$

Then the system $\left\{e_{n}\right\}_{n \in Z}$ forms a basis for $L_{p(\cdot), \rho}$.

Proof In establishing of basis properties of the system (4) in $L_{p(\cdot), \rho}$, we will apply the method of boundary value problems, namely, we will consider the following Riemann problem:

$$
\left\{\begin{array}{l}
F^{+}(\tau)+e^{-2 i \gamma(t)} F^{-}(\tau)=g(\tau) e^{-i \gamma(t),} \\
F^{+} \in H_{p(\cdot), \rho}^{+}, \quad F^{-} \in{ }_{-1} H_{p(\cdot), \rho}^{-},
\end{array}\right.
$$

where $g(\cdot)$ is some Hölder function on $\partial \omega$. We will solve this problem by the method developed in [23]. Here we need some auxiliary functions. Let

$$
(z+1)_{-1}^{\gamma} ; z_{-1}^{\gamma} \quad\left((z-1)_{+1}^{\gamma} ; z_{+1}^{\gamma}\right)
$$

be branches of multi-valued analytic functions

$$
(z+1)^{\gamma} ; z^{\gamma} \quad\left((z-1)^{\gamma} ; z^{\gamma}\right)
$$


which are analytic on the cut along the negative (positive) part of the real axis of the complex plane, respectively. Define

$$
\left(\frac{z+1}{z}\right)_{-1}^{\gamma}=\frac{(z+1)_{-1}^{\gamma}}{z_{-1}^{\gamma}} ; \quad\left(\frac{z-1}{z}\right)_{+1}^{\gamma}=\frac{(z-1)_{+1}^{\gamma}}{z_{+1}^{\gamma}} .
$$

Thus, a particular solution of the problem (8) has the form

$$
\left.\begin{array}{l}
F_{0}^{+}(z)=\frac{1}{2 \pi} \int_{-\pi}^{\pi} \frac{e^{i(\alpha t+\beta \operatorname{sign} t)} g\left(e^{i t}\right) d t}{\left(e^{i t}+1\right)_{-1}^{\gamma_{1}}\left(e^{i t}-1\right)_{+1}^{\gamma_{1}}\left(1-z e^{i t}\right)}(z+1)_{-1}^{\gamma_{1}}(z-1)_{+1}^{\gamma_{2}}, \\
F_{0}^{-}(z)=\frac{1}{2 \pi} \int_{-\pi}^{\pi} \frac{e^{i(\alpha t+\beta \operatorname{signt} g} g\left(e^{i t}\right) d t}{\left(e^{i t}+1\right)_{-1}^{\gamma_{1}}\left(e^{i t}-1\right)_{+1}^{\gamma_{1}}\left(1-z e^{i t}\right)}\left(\frac{z+1}{z}\right)_{-1}^{\gamma_{1}}\left(\frac{z-1}{z}\right)_{+1}^{\gamma_{2}} .
\end{array}\right\}
$$

If the inequality $\left|\gamma_{i}\right|<1, i=1,2$, is fulfilled, from the representation of the function $F_{0}^{ \pm}(z)$ it directly follows that $F_{0}^{+} \in H_{1}^{+} ; F_{0}^{-} \in{ }_{-1} H_{1}^{-}$. From the known relations [21]

$$
\begin{array}{ll}
\int_{-\pi}^{\pi}\left|F_{0}^{+}\left(e^{i t}\right)-F_{0}^{+}\left(r e^{i t}\right)\right| d t \rightarrow 0, & r \rightarrow 1-0 ; \\
\int_{-\pi}^{\pi}\left|F_{0}^{-}\left(e^{i t}\right)-F_{0}^{-}\left(r e^{i t}\right)\right| d t \rightarrow 0, & r \rightarrow 1+0, \\
\int_{-\pi}^{\pi}\left|F_{0}^{-}\left(e^{i t}\right)-F_{0}^{-}\left(r e^{i t}\right)\right| d t \rightarrow 0, & r \rightarrow 1+0,
\end{array}
$$

it follows that

$$
a_{n}^{+}=\frac{1}{2 \pi} \int_{-\pi}^{\pi} F_{0}^{+}\left(e^{i t}\right) e^{i n t} d t, \quad \forall n \in Z_{+} ; \quad a_{k}^{-}=\frac{1}{2 \pi} \int_{-\pi}^{\pi} F_{0}^{-}\left(e^{i t}\right) e^{i k t} d t, \quad \forall k \in N,
$$

where

$$
F_{0}^{+}(z)=\sum_{n=0}^{\infty} a_{n}^{+} z^{n} \quad\left(F_{0}^{-}(z)=\sum_{n=1}^{\infty} a_{n}^{-} z^{-n}\right)
$$

is a Taylor expansion of the function $F_{0}^{+}(z)\left(F_{0}^{+}(z)\right)$ in the neighborhood of zero (an infinitely remote point).

Let us consider the basicity of system $\left\{e_{n}\right\}_{n \in Z}$ in $L_{p(\cdot), \rho}$. Let $g \in L_{p(\cdot), \rho}$ be an arbitrary function. Let us apply Theorem 5 to the non-homogeneous Riemann problem (8). Paying attention to Corollary 1 , we see that if the inequalities

$$
\left\{\begin{array}{l}
-\frac{1}{p\left(\tau_{k}\right)}<\alpha_{k}<\frac{1}{q\left(\tau_{k}\right)}, \quad k=\overline{0, m} ; \\
-\frac{1}{p(0)}<\alpha_{0}+\gamma_{2}<\frac{1}{q(0)} ; \\
-\frac{1}{p(\pi)}<\alpha_{1}+\gamma_{1}<\frac{1}{q(\pi)} ; \\
-\frac{1}{p(\pi)}<\alpha_{m}+\gamma_{1}<\frac{1}{q(\pi)},
\end{array}\right.
$$

are fulfilled, then the problem (8) has a unique solution of the form (9) in classes $H_{p(\cdot), \rho}^{+} \times$ ${ }_{-1} H_{p(\cdot), \rho}^{-}$. Expanding the function $F_{0}^{+}(z)\left(F_{0}^{-}(z)\right)$ in a Taylor series at the point $z=0$ (at the point $z=\infty$ ) we have

$$
F_{0}^{+}(z)=\sum_{n=0}^{\infty} H_{n}^{+}(g) z^{n}, \quad F_{0}^{-}(z)=\sum_{n=1}^{\infty} H_{n}^{-}(g) z^{-n}
$$


Let $F_{0}^{ \pm}(\tau)$ be boundary values on $\partial \omega$ of $F_{0}^{ \pm}(z)$. It is obvious that $F_{0}^{+} \in L_{p(\cdot), \rho}^{+}\left(F_{0}^{-} \in{ }_{-1} L_{p(\cdot), \rho}^{-}\right)$, where $L_{p(\cdot), \rho}^{+}\left({ }_{-1} L_{p(\cdot), \rho}^{-}\right)$is the restriction of class $H_{p(\cdot), \rho}^{+}\left({ }_{1} H_{p(\cdot), \rho}^{-}\right)$on $\partial \omega$. Assume that the following inequalities are true:

$$
-\frac{1}{p\left(\tau_{k}\right)}<\alpha_{k}<\frac{1}{q\left(\tau_{k}\right)}, \quad k=\overline{0, m}
$$

According to the results of [17], the system $\left\{e^{i n t}\right\}_{0}^{\infty}\left(\left\{e^{-i n t}\right\}_{1}^{\infty}\right)$ forms a basis for $L_{p(\cdot), \rho}^{+}$(for $\left.{ }_{-1} L_{p(\cdot), \rho}^{-}\right)$. Expanding the functions $F_{0}^{ \pm}(\tau)$ in these systems we have

$$
F_{0}^{+}\left(e^{i t}\right)=\sum_{n=0}^{\infty} a_{n}^{+} e^{i n t}, \quad F_{0}^{-}\left(e^{i t}\right)=\sum_{n=1}^{\infty} a_{n}^{-} e^{-i n t}
$$

From equations (10) it follows that

$$
a_{n}^{ \pm}=H_{n}^{ \pm}(g), \quad \forall n
$$

Substituting these expansions into (8) we see that the function $g$ can be expanded in a series in $L_{p(\cdot), \rho}$ with respect to the system $\left\{e_{n}\right\}_{n \in Z}$. Lemma 2 implies that such an expansion is unique. So, we have proved the theorem.

Let us consider some special cases of this theorem.

Corollary 2 Let $p \in W L, p^{-}>1$ and $\alpha_{k}=0, k=\overline{2, m-1} ; \beta=0$. If the following inequalities:

$$
\begin{aligned}
& -\frac{1}{p(0)}<\alpha_{0}<\frac{1}{q(0)} ; \quad-\frac{1}{p(\pi)}<\left\{\alpha_{1} ; \alpha_{m}\right\}<\frac{1}{q(\pi)} ; \\
& -\frac{1}{p(\pi)}<\alpha_{1}+2 \alpha<\frac{1}{q(\pi)} ; \quad-\frac{1}{p(\pi)}<\alpha_{m}+2 \alpha<\frac{1}{q(\pi)},
\end{aligned}
$$

hold, then the system of exponents $\left\{e^{i(n+\alpha \operatorname{sign} n) t}\right\}_{n \in Z}$ forms a basis for $L_{p(\cdot), \rho}$.

Consider a more complicated case.

Corollary 3 Let $p \in W L, p^{-}>1$ and $\alpha_{k}=0, k=\overline{2, m-1} ; \alpha=0$. If the inequalities

$$
\begin{aligned}
& -\frac{1}{p(0)}<\alpha_{0}<\frac{1}{q(0)} ; \quad-\frac{1}{p(\pi)}<\left\{\alpha_{1} ; \alpha_{m}\right\}<\frac{1}{q(\pi)} ; \\
& -\frac{1}{p(0)}<\alpha_{0}-\frac{2 \beta}{\pi}<\frac{1}{q(0)} ; \quad-\frac{1}{p(\pi)}<\alpha_{1}+\frac{2 \beta}{\pi}<\frac{1}{q(\pi)} ; \\
& -\frac{1}{p(\pi)}<\alpha_{m}+\frac{2 \beta}{\pi}<\frac{1}{q(\pi)},
\end{aligned}
$$

are fulfilled, then the system of exponents $\left\{e^{i(n t+\beta \operatorname{sign} t \operatorname{sign} n)}\right\}_{n \in Z}$ forms a basis for $L_{p(\cdot), \rho}$.

The authors declare that they have no competing interests. 


\section{Author details}

${ }^{1}$ Nakhchivan State University, University Campus, Nakhchivan, 7012, Azerbaijan. ${ }^{2}$ Department of Non-Harmonic Analysis, Institute of Mathematics and Mechanics of NAS of Azerbaijan, 9 B. Vahabzadeh Str., Baku, 1141, Azerbaijan. ${ }^{3}$ Baku State University, 23 Z. Khalilov Str., Baku, 1148, Azerbaijan.

\section{Acknowledgements}

This work was supported by the Science Development Foundation under the President of the Republic of Azerbaijan Grant No. EIF/GAM-3-2014-6(21)-24/03/1. The authors express their deep gratitude to Professor BT Bilalov, corresponding member of the National Academy of Sciences of Azerbaijan, for his inspiring guidance and valuable suggestions during the work.

Received: 30 May 2015 Accepted: 19 February 2016 Published online: 10 March 2016

\section{References}

1. Paley, R, Fourier, WN: Transforms in the Complex Domain. Amer. Math. Soc. Collog. Publ., vol. 19. Am. Math. Soc., Providence (1934)

2. Bilalov, BT: Basicity of some systems of exponents, cosines and sines. Differ. Uravn. 26(1), 10-16 (1990)

3. Bilalov, BT: On basicity of systems of exponents, cosines and sines in $L_{p}$. Dokl. Ross. Akad. Nauk 365(1), 7-8 (1999)

4. Bilalov, BT: On basicity of some systems of exponents, cosines and sines in $L_{p}$. Dokl. Ross. Akad. Nauk 379(2), 7-9 (2001)

5. Bilalov, BT: Bases of exponents, cosines and sines which are eigenfunctions of differential operators. Differ. Uravn. 39(5), 1-5 (2003)

6. Young, RM: An Introduction to Nonharmonic Fourier Series. Springer, Berlin (1980)

7. Christensen, O: An Introduction to Frames and Riesz Bases. Birkhäuser Boston, Boston (2003)

8. Sedletskii, AM: Classes of Analytic Fourier Transforms and Exponential Approximations. Fizmatlit, Moscow (2005)

9. Duffin, RJ, Schaeffer, AC: A class of no harmonic Fourier series. Trans. Am. Math. Soc. 72(341), 341-366 (1952)

10. Heil, C: A Basis Theory Primer. Springer, Berlin (2011)

11. Kovacik, O, Rakosnik, J: On spaces $L^{p(\cdot)}$ and $W^{k, p(\cdot)}$. Czechoslov. Math. J. 41(116), 592-618 (1991)

12. Fan, $X$, Zhao, D: On the spaces $L^{p(x)}(\Omega)$ and $W^{m, p(\cdot)}(\Omega)$. J. Math. Anal. Appl. 263, 424-446 (2001)

13. Kokilashvili, $\vee$, Paatashvili, V: On Hardy classes of analytic functions with a variable exponent. Proc. A. Razmadze Math. Inst. 142, 134-137 (2006)

14. Sharapudinov, II: Some problems in approximation theory in the spaces $L^{p(x)}(E)$. Anal. Math. 33(2), 135-153 (2007)

15. Kokilashvili, V, Samko, S: Singular integrals in weighted Lebesgue spaces with variable exponent. Georgian Math. J. 10(1), 145-156 (2003)

16. Cruz-Uribe, DV, Fiorenza, A: Variable Lebesgue Spaces: Foundations and Harmonic Analysis. Springer, Berlin (2013)

17. Bilalov, BT, Guseynov, ZG: Basicity of a system of exponents with a piece-wise linear phase in variable spaces. Mediterr. J. Math. 9(3), 487-498 (2012)

18. Bilalov, BT, Guseynov, ZG: Basicity criterion for perturbed systems of exponents in Lebesgue spaces with variable summability. Dokl. Ross. Akad. Nauk 436(5), 586-589 (2011)

19. Bilalov, BT, Guseynov, ZG: Bases from exponents in Lebesque spaces of functions with variable summability exponent. Trans. NAS Azerb. 27(1), 43-48 (2008)

20. Ismayilov, NA, Bases, NNP: Of exponents in weighted Hardy classes. Int. J. Math. Sci. Eng. Appl. 7(3), 101-109 (2013)

21. Danilyuk, II: Irregular Boundary Value Problems in the Plane. Nauka, Moscow (1975)

22. Najafov, TI, Nasibova, NP: On the Noetherness of the Riemann problem in a generalized weighted Hardy classes. Azerb. J. Math. 5(2), 109-124 (2015)

23. Gakhov, FD: Boundary Value Problems. Nauka, Moscow (1977)

\section{Submit your manuscript to a SpringerOpen ${ }^{\circ}$ journal and benefit from:}

- Convenient online submission

Rigorous peer review

- Immediate publication on acceptance

Open access: articles freely available online

- High visibility within the field

- Retaining the copyright to your article 